\title{
An Appropriate Modulation Scheme for High Density Visible Light Communicaton System
}

\author{
Chenzhou Lin', Yijun Zhu ${ }^{2}$ and Yanyu Zhang ${ }^{3}$ \\ School of The PLA information engineering university, Zhengzhou 450000, China \\ xdlinchenzhou@126.com
}

Keywords: SC-PPM; high density; visible light communication; energy efficient.

\begin{abstract}
An appropriate modulation scheme SC-PPM is considered in this article for the high density visible light communication (VLC) system. Compared with OOK, SC-PPM is a more power efficient and reliable scheme and it can achieve the requirement for both illumination and communication. An optimization problem is formulated to reduce the total transmitted energy produced by LED lamps while satisfying the desired illuminance level and communication quality index. the simulation result show that SC-PPM can save the energy consuming compared with traditional PPM.
\end{abstract}

\section{Introduction}

Currently, LEDs are popularly employed in our daily life due to various advantages. With the popularity of LED lamps, visible light communication based on the LED lamps will face with a unprecedented development opportunity. According to prediction, there will be millions of LED lamps in our daily illumination and a huge visible light communication network is gradually formed.

Advanced modulation formats are becoming increasingly important in the high density VLC system. Due to the advantage of simple on-off switching controlling, OOK modulation can be easily used in visible light communication[1]. However, lighting plays a key role in LED-based VLC, the possible a series of sequence of consecutive zeros or ones will cause an unbalanced optical power on LED lighting.PPM is a good way to resist the unsteady illumination caused by OOK, it is widely employed due to the advantage of good power efficiency and low bit error rate[2,3].

In the high density VLC system, the electric energy consumption produced by LEDs will become an important index to measure the performance of VLC system. While the illumination is a key contribution to electric energy consumption of LED lamps, as the function of communication adds to LED lamps, it will consume more energy than lighting alone. Therefore, the influence of VLC energy consumption should be taken into consideration.

Recently, SC-PPM has been studied to combine communication with illumination. Compared with PPM,SC-PPM transmits optical signal by using sub-carrier of frequency $28.8 \mathrm{kHz}[4]$ and it is a more reliable scheme because it can get rid of the influence of background light effect produced by LED lamp[5]. In the SC-PPM scheme, an energy optimization problem is formulated. We can change the modulation depth of SC-PPM to achieve the optimization of the LED lighting and make it a energy efficient way according to the required illumination level and communication quality.

The rest of this letter is organized as follow. In section II, we compare the transmission capacity and bit error rate (BER) between SC-PPM and OOK. In Section III, a method of optimization of LED power consumption is taken into account and simulation result is presented. Conclusion is drawn in section IV. 


\section{Modulation scheme performance}

\section{1.structure of SC-PPM}

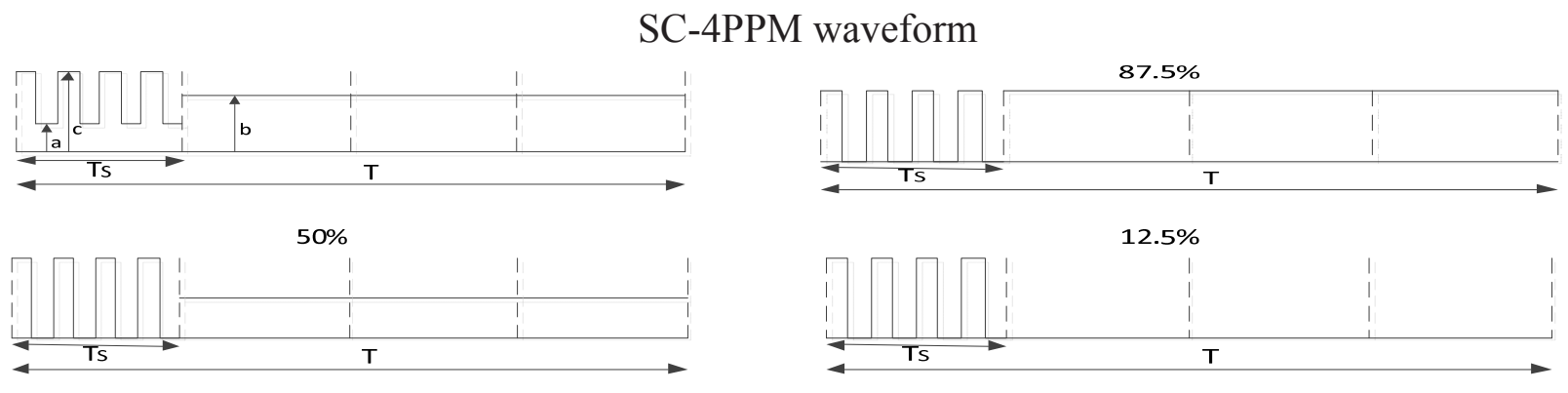

Fig. 1. SC-4PPM signal waveform

This article considers an SC-PPM scheme for modulation in the high density VLC system. The structure of the SC-PPM is divided into two components, including the subcarrier part and a DC part. Taking an example of SC-4PPM, a transmitted symbol is divided into four intervals. The symbol value depends on the position of sub-carrier, the other three slots have a constant DC amplitude . By changing the value of $a, b, c$, the brightness index can vary from $0 \%$ to $100 \%$.

\section{Required bandwidth}

Using bit rate to measure the capacity between SC-PPM and OOK and make a comparison under the condition of the same slot width. If a slot width is $T_{S}$, the capacity of OOK $R_{b}$ is $1 / T_{S}$. If a symbol is $\mathrm{N}$ bit, the length of symbol is $2^{\mathrm{N}} \mathrm{T}_{\mathrm{S}}$, the capacity of $\mathrm{SC}-\mathrm{PPM}$ is $\mathrm{R}_{\mathrm{b}}{ }_{\mathrm{b}}=\mathrm{N} /\left(2^{\mathrm{N}} \mathrm{T}_{\mathrm{S}}\right)$. The ideal low-pass bandwidth is $\mathrm{B}=\mathrm{R}_{\mathrm{b}} / 2$. Therefore, under the condition of the same bit rate, the required bandwidth ratio for OOK and SC-PPM is $B / B^{\prime}=2^{M} / M$.

\section{Bit error rate}

The bit error rate for OOK-coded optical signal, detected with a photodiode, can be expressed as a function [6]:

$$
B E R_{\text {оок }}=\frac{1}{2} \operatorname{erfc}\left(\frac{1}{2 \sqrt{2}} \sqrt{S N R}\right) \ldots \ldots \text { (1) }
$$

Under the Gaussian noise channel, the bit error rate for PPM can be expressed as a function;

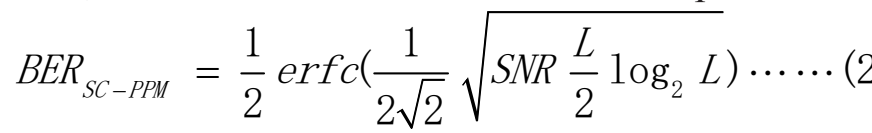

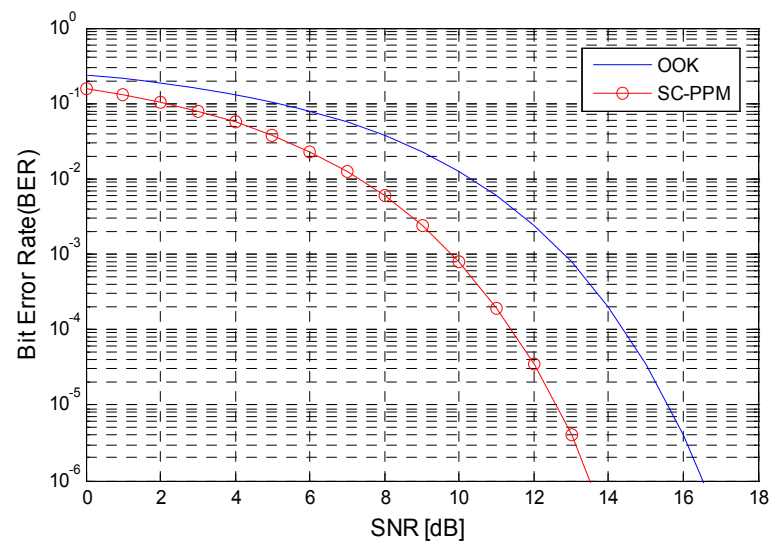

Fig. 1.Bit Error Rate versus SNR plot of OOK and SC-PPM

\section{Energy optimization}

\section{Illuminance and optical power produced by LED}

As for SC-4PPM scheme, the amplitude of optical signal is (c-a), the amplitude of DC part is b. In the indoor VLC system, as shown in Fig.4,the light source of the transmitter is white LED, The 
luminous flux produced by LED lamp can be represented by[7]:

$$
\Phi_{i}=\left[\left(a_{i}+c_{i}\right) * \frac{T_{S}}{2}+b * 3 T_{S}\right] \times \Phi_{\text {max }}
$$

By changing the value of $a, b, c$. the brightness index can be controlled from $0 \%$ to $100 \%$, $\Phi$ max is the maximum luminous flux produced by LED lamp, it is obtained under the condition of $\mathrm{a}=\mathrm{b}=\mathrm{c}=\mathrm{c}_{\max }$. When a source with Lambert radiation characteristic is assumed and the received illuminance at workplace $\mathrm{j}$ from LED $\mathrm{i}$ is modeled as[8]:

$$
\begin{aligned}
& e_{i j}=I_{0} \cos ^{m} \theta \cos \psi / r^{2} \cdots \cdots(4) \\
& I_{0}=(\mathrm{m}+1) \Phi_{i} / 2 \pi \cdots \cdots(5)
\end{aligned}
$$

$\theta$ is the irradiance angle of light source, $\psi$ is the incidence angle of PD, $r$ represents the distance between LED and PD. $m$ is the Lambert index.

The total illuminance at workplace $\mathrm{j}$ from different LED lamps can be represented by:

$$
E_{j}=\sum_{i=1}^{L} e_{i j} \cdots \cdots(6)
$$

In SC-PPM, the transmitted signal depends on the position of the sub-carrier, therefore, the transmitted optical power only considers the sub-carrier part and signal strength can be computed as.

$$
P_{i}^{t}=(c-a) \times P_{\max }^{t}
$$

$\mathrm{P}_{\mathrm{i}}^{\mathrm{t}}$ is the optical power transmitted by the $\mathrm{i}^{\text {th }}$ LED and $\mathrm{P}_{\max }^{\mathrm{t}}$ is the maximum optical power.

The received power at PD can be defined as:

$$
P_{r}=\sum_{i=1}^{L}\left(H(0) \times P_{i}^{t}\right) \cdots \cdots
$$

$\mathrm{H}(0)$ is the channel DC gain and $\mathrm{P}_{\mathrm{r}}$ is the total received optical power at workplace $\mathrm{j}$. When we assume Lambertian radiation transmitter, $\mathrm{H}(0)$ is given by:

$$
H(0)=\frac{A_{r}(m+1)}{2 \pi r^{2}} \cos ^{m}(\theta) \cos \varphi \cdots \cdots(9)
$$

The bit rate $\mathrm{R}_{\mathrm{b}}$ under the wireless optical link is related to the electrical SNR[9]:

$$
S N R=\frac{R^{2} P_{r}^{2}}{R_{b} N_{0}}=\frac{R^{2} H^{2}(0) P_{t}^{2}}{R_{b} N_{0}} \cdots \cdots
$$

$\mathrm{R}$ is the photodiode responsivity, $\mathrm{N}_{0}$ is the power spectrum density of additive white Gaussian noise(AWGN).According to (2)(10), the BER of SC-PPM can be represented by:

$$
B E R_{S C-P P M}=\frac{1}{2} \operatorname{erfc}\left(\frac{1}{2 \sqrt{2}} \sqrt{\frac{\mathrm{L}_{r}^{2} \log _{2} L}{2 R_{b} N_{0}}}\right) \ldots \ldots
$$

\section{Optimization formulation}

Therefore, to achieve a required value of luminance flux, we try to minimize the total power consumption produced by LED lamps. As the luminance flux produced by LED lamps is proportional to the transmitted power, namely, the objective is to minimize the total luminance flux of LED lamps.

$$
\text { Minimize } \sum_{i=1}^{M}\left(\frac{T_{S}}{2} \times\left(\mathrm{a}_{i}+c_{i}\right)+3 T_{S} \times b_{i}\right) \times \Phi_{\max }
$$

Subject to

$$
\begin{aligned}
& \sum_{i=1}^{M}\left(\frac{T_{S}}{2} \times\left(\mathrm{a}_{i}+c_{i}\right)+3 T_{S} \times b_{i}\right) \times \frac{\Phi_{\max }(m+1)}{2 \pi r^{2}} \cos ^{m}(\theta) \cos \varphi \geq E_{j}^{r} \ldots \ldots \\
& \sum_{i=1}^{M}\left(c_{i}-a_{i}\right) \times P_{\max }^{t} \frac{A_{r}(m+1)}{2 \pi r^{2}} \cos ^{m}(\theta) \cos (\varphi) \geq P_{r}^{\min } \ldots \ldots(14)
\end{aligned}
$$


$\mathrm{P}^{\mathrm{min}} \mathrm{r}$ is the minimum received power according to the equation (11) when BER and $\mathrm{R}_{\mathrm{b}}$ is given in a VLC system. Equation (13) (14) gives the constraints for required luminance level and communication performance.

Assuming that the simulation environment is in a indoor room. There are four LED lamps on the ceiling, the received workplace $\mathrm{j}$ located at the center place of the floor. the simulation parameters are the follows.

Table 1 Simulation Parameters

\begin{tabular}{lcc}
\hline Name & Symbol & Value \\
\hline Bit rate & $\mathrm{R}_{\mathrm{b}}$ & $10^{6} \mathrm{bps}$ \\
Single LED power & $\mathrm{P}_{\max }$ & $20 \mathrm{~mW}$ \\
semi-angle at half power & $\psi_{1 / 2}$ & $60 \mathrm{deg}$ \\
Center luminous intensity & $\mathrm{I}$ & $730 \mathrm{~cd}$ \\
Detected area & $\mathrm{Ar}$ & $1 \mathrm{~cm}^{2}$ \\
AWGN & $\mathrm{N}_{0}$ & $10^{-21}$ \\
Photodiode responsivity & $\mathrm{R}$ & 0.5 \\
Incidence angle & $\theta$ & $120 \mathrm{deg}$ \\
Required luminance & $\Phi$ req & $4001 \mathrm{ux}$ \\
\hline
\end{tabular}

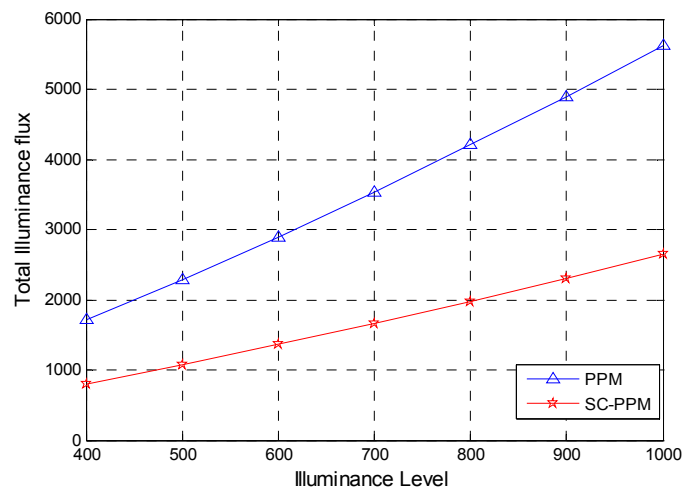

Fig. 3.Total illuminance flux produced by LEDs under the illuniance level at 400 to 1000lux of PPM and SC-PPM

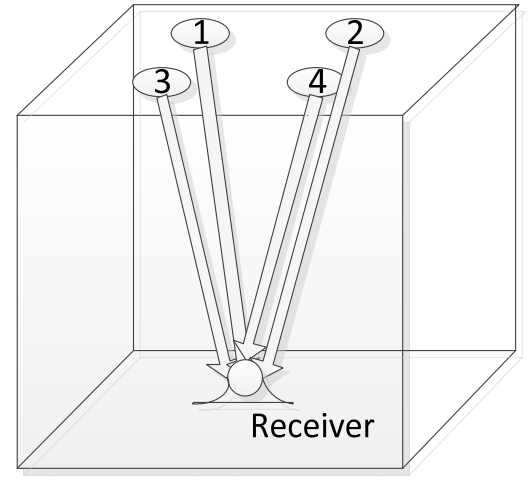

Fig. 4 system model for. indoor communication

The simulation demonstrate the minimum total transmitted optical power produced by all LED lamps for different required illuminance level. The proposed scheme SC-PPM compared with the traditional PPM (the amplitude of waveform is $a=c=1, b=0$ ).fig 2 shows the energy saving using the proposed scheme under the condition of required illuminance level 400lux-1000lux.in a high density VLC system, the proposed scheme SC-PPM reduces the total optical power produced by LED lamps and achieves the improvement in illuminance energy saving. it is an energy-efficient modulation scheme compared with other schemes for satisfying the requirements for both illuminance and communication.

\section{Conclusion}

This paper proposed an appropriate and reliable modulation scheme. An optimization problem is formulated to reduce the total illumination energy produced by LED lamps while satisfying the desired brightness and communication quality. Simulation result demonstrated that SC-PPM can save energy consuming in the high density VLC system .

\section{References}

[1] C. Eunbyeol et al., "NRZ-OOK Signaling with LED Dimmingfor Visible Light Communication Link," Proc. 16th Euro.Conf. Networks and Optical Commun., 2011, p. 32-35.

[2] Nazmy Azzam, Moustafa H. Aly and A.K. AbouiSeoud"Bandwidth and Power Efficiency ofVarious PPM Schemes for Indoor Wireless Optical Communications." National Radio Science 
Conference, 2009. New Cairo ,National17-19 March 2009. p.0-11

[3] Ghafour Amouzad Mahdiraji and Edmond Zahedi Comparison of Selected Digital Modulation Schemes (OOK, PPM and DPIM) for Wireless Optical Communications. Research and 4th Student Conference on Research and Development, 2006. SCOReD 2006. Selangor, 27-28 June 2006 , p.5-10

[4] Hidemitsu Sugiyama, Shinichiro Haruyama, and Masao Nakagawa"Brightness Control Methods for Illumination and Visible-Light Communication Systems.' ' Wireless and Mobile Communications, 2007. ICWMC '07. Third International Conference on. Guadeloupe,4-9 March 2007.p.78

[5] Chi Nan, "LED visible light communication technology" Tsinghua University Press,2013,p,67

[6] Taissir Y. Elganimi Tripoli, Libya, "Studying the BER Performance, Power- and BandwidthEfficiency for FSO Communication Systems under Various Modulation Schemes.” Applied Electrical Engineering and Computing Technologies (AEECT), 2013 IEEE Jordan Conference on,Amman,3-5 Dec. 2013,p.1-6

[7] Irfanud Din and Hoon Kim, "Performance Analysis of SC-L-PPM for energy efficient visible light communication ." Consumer Electronics (ISCE 2014), The 18th IEEE International Symposium on, JeJu Island, 22-25 June 2014,p, 1 - 2.

[8] J. Grubor, O. C. G. Jamett, J. W. Walewski, S. Randel, and K.-D. Langer"High-speed wireless indoor communication via visible light," in Proc.ITG Bachbericht, 2007, p. 1-5.

[9] Hidemitsu Sugiyama, Shinichiro Haruyama, and Masao Nakagawa“Brightness Control Methods for Illumination and Visible-Light Communication Systems.', Wireless and Mobile Communications, 2007. ICWMC '07. Third International Conference on. Guadeloupe,4-9 March 2007.p.78 